Proceedings of the Edinburgh Mathematical Society (2004) 47, 191-204 (C)

DOI:10.1017/S0013091503000245 Printed in the United Kingdom

\title{
PROPAGATION OF SMALLNESS FOR SOLUTIONS OF GENERALIZED CAUCHY-RIEMANN SYSTEMS
}

\author{
E. MALINNIKOVA \\ Department of Mathematics, Norwegian University of Science and Technology, \\ N-7491, Trondheim, Norway (eugenia@math.ntnu.no)
}

(Received 25 March 2003)

\begin{abstract}
Let $u$ be a solution of a generalized Cauchy-Riemann system in $\mathbb{R}^{n}$. Suppose that $|u| \leqslant 1$ in the unit ball and $|u| \leqslant \varepsilon$ on some closed set $E$. Classical results say that if $E$ is a set of positive Lebesgue measure, then $|u| \leqslant C \varepsilon^{\alpha}$ on any compact subset of the unit ball. In the present work the same estimate is proved provided that $E$ is a subset of a hyperplane and the (capacitary) dimension of $E$ is greater than $n-2$. The proof gives control of constants $C$ and $\alpha$.
\end{abstract}

Keywords: Generalized Cauchy-Riemann system; Riesz capacity; real-analytic functions; quantitative continuation

2000 Mathematics subject classification: Primary 31B35

Secondary 35B35; 35J45

\section{Introduction}

Given a subset $E$ of the unit ball $B \subset \mathbb{R}^{n}$ and a class of (vector) functions $\mathcal{A} \subset C(B)$, we say that $E$ is a propagation of smallness set for $\mathcal{A}$ if for any $u \in \mathcal{A}$

$$
|u(x)| \leqslant C\|u\|_{B}^{1-\alpha}\|u\|_{E}^{\alpha},
$$

where $\alpha=\alpha(x, E)>0$ is independent of $u$ and bounded away from 0 on compact subsets of $B$. We will consider this problem for $\mathcal{A}$ being the space of (real) analytic functions or the space of solutions to some differential equation.

Propagation of smallness was intensively studied for solutions of second-order elliptic equations. In particular, if $E$ is a small ball, then (1.1) is an immediate consequence of the three-balls theorem. Various versions of this theorem for solutions of second-order elliptic equations were proved by Gusarov and Landis [11], and, using another approach, by Brummelhuis [5]. The result due to Korevaar and Meyers [10] shows that when $\mathcal{A}$ is the space of harmonic functions and $E$ is a ball one can take $C=1$ in (1.1). The case of a set $E$ of positive Lebesgue measure was investigated independently by Nadirashvili and Vessella. It is proved in $[\mathbf{1 5 , 2 0}]$ that if $\mathcal{A}$ is the space of real analytic functions that have analytic extension to a fixed domain in $\mathbb{C}^{n}$ (for example, $\mathcal{A}$ is a space of solutions to an elliptic equation with analytic coefficients) and $m(E)>0$, then (1.1) holds. For 
arbitrary second-order elliptic equations the question of propagation of smallness from a set of positive measure is open. Interesting inequalities are proved in $[\mathbf{1 6}, \mathbf{2 1}]$, but they are weaker than (1.1).

In this paper $\mathcal{A}$ is the space of solutions to a generalized Cauchy-Riemann (GCR) system. More precisely, we consider systems of first-order differential equations with constant coefficients that are factors of the Laplacian. The simplest example is the classical Cauchy-Riemann system - for this case, $\mathcal{A}$ is the class of functions analytic in the unit disc. It can be proved that $E$ is a propagation of smallness set for analytic functions if and only if $E$ is non-polar (see $\S 2$ ). The author is grateful to Vladimir Eiderman for pointing out this result. It is related to the fact that the logarithm of the module of an analytic function is subharmonic (and harmonic off the zero set of the function). In other words, we have a description of the space of modules of analytic functions. Unfortunately, there is no such description for modules of solutions to GCR systems. It is well known (see [19]) that if $u$ is a solution to a GCR system, then $|u|^{p}$ is subharmonic for some $p<1$, where $p$ depends on the system. Being a partial substitution to the subharmonicity of the logarithm, this result is difficult to use in our problem. The difference between analytic functions and solutions to higher-dimensional systems is discussed in the remarkable work of Wolff [22]. It shows that solutions of (even the simplest) generalization of Cauchy-Riemann systems do not share a number of important properties of analytic functions.

We formulate a condition that is sufficient for propagation of smallness for GCR systems. It is clear that zero sets of $\mathcal{A}$ are of dimension at most $n-2$. We assume that $E$ is a subset of a hyperplane and its (capacitary) dimension is greater than $n-2$. Then $E$ is a propagation of smallness set for $\mathcal{A}$ and we have estimates on $\alpha$ in (1.1).

Main Result. Let $A$ be a differential operator with constant coefficients, $A=$ $\sum_{1}^{n} A_{j} \partial_{j}$, that is a factor of the Laplacian. Let $\mathcal{A}$ be the space of solutions to $A u=0$, where $u: B \rightarrow \mathbb{R}^{m}$. Suppose that $E$ is a compact subset of a hyperplane, $E \subset B_{1 / 2}$, and for some $\delta>0$ the Riesz capacity $C_{n-2+\delta}(E)=\mathcal{C}$ is positive (for the definition of the capacity see (5.3) below). Then (1.1) holds with some $C=C(n)$ and $\alpha=\alpha(x, n, A, \delta, \mathcal{C})$ bounded away from 0 on compact subsets of $B$.

The paper is organized as follows. In $\S 2$ we give a simple proof of (1.1) for analytic functions. The proof is based on the definition of capacity via Chebyshev constants and gives an estimate for $\alpha$ in terms of the logarithmic capacity. This result is applied in $\S 3$ to obtain propagation of smallness for real-analytic functions in $\mathbb{R}^{N}$. The following result of $\S 3$ is of independent interest.

Key Lemma. Let $A\left(B_{R}\right)$ be the set of real analytic functions $f$ on $B_{R}$ that admit analytic continuation $\tilde{f}$ to the closed complex ball $B_{R, \mathbb{C}}=\left\{z \in \mathbb{C}^{N}:|z| \leqslant R\right\}$ such that $|\tilde{f}| \leqslant 1$ on $B_{R, \mathbb{C}}$. Let $K \subset B_{r}$ be a compact set of positive Riesz $(N-1+\delta)$-capacity. Then

$$
\sup _{B_{r}}|f| \leqslant \sup _{K}|f|^{\alpha},
$$

for any $f \in A\left(B_{R}\right)$, where $\alpha=\alpha\left(N, \delta, r^{1-N-\delta} C_{N-1+\delta}(K)\right)$. 
We use this lemma to prove the Main Result in $\S 4$, which begins with definitions and examples of GCR systems and factors of the Laplacian. In the last section, notions and results from potential theory are collected.

We use $N$ to denote the dimension of the real (complex) space when working with (real-)analytic functions. Then in $\S 4$ we change the notation and use $n$ for the dimension of the space where a GCR system is considered. The reason is that in this section the results on analytic functions in smaller-dimensional spaces are used.

Throughout the paper $c(N), c(n), \ldots$ stand for different constants depending only on $N$, n, etc. The values of these constants changes from line to line and is of no importance for us. By $c_{l}(N), l=0,1, \ldots$, we denote constants that are fixed through the paper. We use the standard notation $\mathcal{H}^{s}$ for the Hausdorff measures and use $m$ to denote the Lebesgue measure.

\section{Propagation of smallness for analytic functions}

\subsection{Formulation of the result}

In this section we consider analytic functions of one complex variable. Suppose that $f$ is analytic and bounded by 1 in the ball $B_{R}=\{|z|<R\}$ and suppose that $|f| \leqslant \varepsilon$ on a compact subset $K \subset B_{r}=\{|z|<r\}$, where $r<R$. We consider estimates of the type

$$
\sup _{B_{r}}|f| \leqslant C \varepsilon^{\alpha}, \quad \text { for some } \alpha=\alpha(R, r, K) \text { and an absolute constant } C .
$$

It is clear that such estimates hold with some $\alpha$ and $C$ if and only if $K$ is a non-polar set. Then one can take $C=1$ and $\alpha=\max _{B_{r}} \omega\left(K, B_{R}, z\right)$, where

$$
\omega\left(K, B_{R}, z\right)=\inf \left\{u(z), u \text { is superharmonic in } B_{R}, u \geqslant 0 \text { on } B_{R} \text { and } u \geqslant 1 \text { on } K\right\}
$$

is the harmonic measure of $K$ relative to $B_{R}$. Our aim is to estimate $\alpha$ in terms of the logarithmic capacity of $K$. Though the question seems to be classical, we failed to find the reference in classical monographs on potential theory. For this reason, we give an elementary proof of the following statement.

Theorem 2.1. Suppose that $4 r<R<1$ and $f$ and $K$ are as above. If $K$ is not a polar set, then (2.1) holds with $C=1$ and

$$
\alpha=\frac{1}{8}\left(\ln \frac{3 r}{C_{0}(K)}\right)^{-1}
$$

where $C_{0}(K)$ is the logarithmic capacity of $K$ (see (5.1) below).

Remark 2.2. As is mentioned in the last section, $C_{0}$ is a non-decreasing function on compact sets and $C_{0}(K) \leqslant C_{0}\left(B_{r}\right)=r$, thus the expression above is well defined. 


\subsection{Proof of Theorem 2.1}

We fix $m$ to be the greatest integer such that

$$
\varepsilon\left(\frac{3 r}{C_{0}(K)}\right)^{m} \leqslant 1
$$

Then we have the following inequalities for $m$ :

$$
m \leqslant \frac{-\ln \varepsilon}{\ln (3 r)-\ln C_{0}(K)} \leqslant \frac{\delta^{-1} \varepsilon^{-\delta}}{\left(\ln (3 r)-\ln C_{0}(K)\right)} .
$$

The last inequality is valid for any $\delta>0$ and is easy to check.

Now, let $z_{0}, z_{1}, \ldots, z_{m} \in K$ be such that

$$
d_{m}(K)=\left(\prod_{0 \leqslant j<k \leqslant m}\left|z_{j}-z_{k}\right|\right)^{2 / m(m+1)}
$$

is the $m$ th transfinite diameter of $K$ (see $\S 5.2$ ). We consider the Lagrange interpolation polynomial for $f$ with the nodes $z_{0}, z_{1}, \ldots, z_{m}$ :

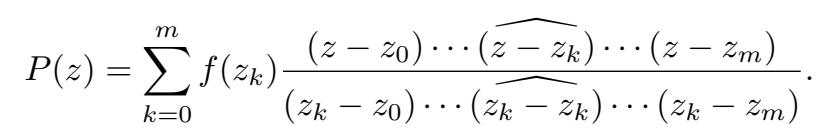

First, we note that for any $z \in K$ the product of the pairwise distances between $z_{0}, \ldots, z_{k-1}, z, z_{k+1}, \ldots, z_{m}$ is less than or equal to the same product for $z_{0}, z_{1}, \ldots, z_{m}$. Hence,

$$
\left(z_{k}-z_{0}\right) \cdots\left(\widehat{z_{k}-z_{k}}\right) \cdots\left(z_{k}-z_{m}\right)=\max _{z \in K}\left(z-z_{0}\right) \cdots\left(\widehat{z-z_{k}}\right) \cdots\left(z-z_{m}\right) .
$$

Thus the right-hand side of the above inequality is greater than or equal to $\rho_{m}^{m}$, where $\rho_{m}$ is the $m$ th Chebyshev constant for $K$ (see $\S 5.2$ for the definition). And, using an elementary estimate $\rho_{m} \geqslant C_{0}(K)$ (see (5.2) below), we get

$$
\left(z_{k}-z_{0}\right) \cdots\left(\widehat{z_{k}-z_{k}}\right) \cdots\left(z_{k}-z_{m}\right) \geqslant C_{0}(K)^{m}
$$

for any $k$. Thus, we get an estimate for the interpolation polynomial on $B_{r}$ :

$$
\max _{B_{r}}|P(z)| \leqslant(m+1) \varepsilon\left(\frac{2 r}{C_{0}(K)}\right)^{m} .
$$

Using the integral formula for the remainder of the Lagrange interpolation, we get the following inequality for $z \in B_{r}$ :

$$
|f(z)-P(z)|=\left|\frac{1}{2 \pi \mathrm{i}} \int_{|\zeta|=R} \frac{f(\zeta)\left(z-z_{0}\right) \cdots\left(z-z_{m}\right)}{(\zeta-z)\left(\zeta-z_{0}\right) \cdots\left(\zeta-z_{m}\right)} \mathrm{d} \zeta\right| \leqslant\left(\frac{2 r}{R-r}\right)^{m} .
$$


We now combine estimates (2.4) and (2.5), and use (2.2):

$$
\begin{aligned}
\max _{B_{r}}|f| & \leqslant(m+1) \varepsilon\left(\frac{2 r}{C_{0}(K)}\right)^{m}+\left(\frac{2 r}{R-r}\right)^{m} \\
& \leqslant\left((m+1) \varepsilon\left(\frac{3 r}{C_{0}(K)}\right)^{m}+1\right)\left(\frac{2}{3}\right)^{m} \\
& \leqslant \frac{3}{2}(m+2) \varepsilon^{\beta}, \quad \text { where } \beta=\frac{\ln \frac{3}{2}}{\ln (3 r)-\ln \left(C_{0}(K)\right)} .
\end{aligned}
$$

Then we apply (2.3) with $\delta=\frac{2}{3} \beta$ to get

$$
\max _{B_{r}}|f| \leqslant 10 \varepsilon^{\beta / 3} .
$$

So (2.1) is proved with $C=10$ and $\alpha=\frac{1}{3} \beta$. Now, applying the theorem to functions $f^{M}$, where $M$ is any positive integer, we see that if (2.1) is valid with some absolute constant, then it is valid with $C=1$, and we are done.

\subsection{Corollaries and remarks}

Clearly, if $K$ has a positive measure, it is non-polar and we can apply the theorem. Later we need the following result.

Corollary 2.3. Let $f$ and $K$ be as in the theorem. Suppose that $K$ is a subset of a line and it has a positive linear measure $|K|$. Then estimate (2.1) is satisfied with

$$
\alpha=\frac{1}{8}\left(\ln \frac{12 r}{|K|}\right)^{-1}
$$

Proof. Theorem 2.1 combined with the classical inequality $C_{0}(K) \geqslant \frac{1}{4}|K|$ (here we use the assumption that $K$ is a subset of a line (see $\S 5.1)$ ) implies the above estimate.

If we fix $r$ and $R$, then $\alpha$ in the theorem can be estimated by the Wiener capacity of $E$ (for the definition see $\S 5.1$ ).

Corollary 2.4. Let $r<R<1$ be some positive numbers and let $K$ be a compact subset of $B_{r}$. Then for any function $f$ analytic in $B_{R}$,

$$
\sup _{B_{r}}|f| \leqslant\left(\sup _{K}|f|\right)^{\alpha}\left(\sup _{B_{R}}|f|\right)^{1-\alpha}, \quad \text { where } \alpha \geqslant c(r, R) \operatorname{cap}(K) .
$$

Proof. First suppose that $4 r<R$. Then, applying the theorem, we get the required inequality with

$$
\alpha=\frac{1}{8}\left(\ln \frac{3 r}{C_{0}(K)}\right)^{-1}=\frac{1}{8}\left(\ln 3 r+\operatorname{cap}(K)^{-1}\right)^{-1} \geqslant c(r) \operatorname{cap}(K) .
$$

If $4 r>R$, we set $s=\frac{1}{4}(R-r)$ and take a ball $B(a, s)$ such that $B(a, 4 s) \subset B_{R}$ and $\operatorname{cap}(E) \geqslant c(r, R) \operatorname{cap}(K)$, where $E=K \cap B(a, s)$. Then, using the theorem, we get

$$
\sup _{B(a, s)}|f| \leqslant\left(\sup _{K}|f|\right)^{\alpha}\left(\sup _{B_{R}}|f|\right)^{1-\alpha}, \quad \text { where } \alpha \geqslant c(s) \operatorname{cap}(E) \geqslant c(r, R) \operatorname{cap}(K) .
$$


We need to replace $B(a, s)$ by $B_{r}$. The standard procedure is to take a chain of balls and apply Hadamard's three-circle theorem at each step. Or a continuous version of this 'propagation of smallness' due to Arakelian and Shahgholian [3] can be used.

Recently, an estimate between relative capacity and condenser capacity for compact subsets of $\mathbb{C}^{N}$ was proved by Siciak $[\mathbf{1 8}]$. Together with $[\mathbf{2}, \mathbf{4}, \mathbf{1 3}]$, it gives a quantitative relation between the logarithmic capacity (or transfinite diameter) and the condenser capacity. Theorem 2.1 can be obtained from these multidimensional results. In the onedimensional case we use elementary estimates and interpolation and approximation by polynomials. We end this section by proving an estimate for $\alpha$ from above.

Remark 2.5. Suppose that inequality (2.1) is satisfied with some constant $C$. Let $P_{m}^{*}$ be an extremal polynomial of degree $m$ for $K$, i.e. the one that gives the $m$ th Chebyshev constant. It is clear that all roots of $P_{m}^{*}$ lie in $B_{r}$. Thus we have $\max _{B_{R}}\left|P_{m}^{*}\right| \leqslant(R+r)^{m}$ and $\max _{K}\left|P_{m}^{*}\right|=\rho_{m}(K)^{m}$. Using (2.1), we get

$$
\rho_{m}^{m}\left(B_{r}\right) \leqslant \max _{B_{r}}\left|P_{m}^{*}\right| \leqslant C(R+r)^{m(1-\alpha)} \rho_{m}(K)^{m \alpha} .
$$

Taking the $m$ th root, we obtain the inequality for the limits

$$
r \leqslant(R+r)^{1-\alpha} C_{0}(K)^{\alpha} .
$$

This implies

$$
\alpha \leqslant \frac{\ln (R+r)-\ln r}{\ln (R+r)-\ln \left(C_{0}(K)\right)} \leqslant \frac{\operatorname{cap}(K)}{\operatorname{cap}(K) \ln r+1} \ln \left(1+\frac{R}{r}\right) .
$$

In particular, $\operatorname{cap}(K)>0$, and we have an estimate for $\alpha$ from above.

\section{Propagation of smallness for real analytic functions in $\mathbb{R}^{N}$}

\subsection{Introductory remarks}

We denote by $A\left(B_{R}\right)$ the set of all real analytic functions $f$ on $B_{R} \subset \mathbb{R}^{N}$ that have analytic continuation $\tilde{f}$ to the closed complex ball $B_{R, \mathbb{C}}=\left\{z \in \mathbb{C}^{N}:|z| \leqslant R\right\}$ such that $|\tilde{f}| \leqslant 1$ on $B_{R, \mathbb{C}}$.

Let $K$ be a compact subset of $B_{r}$, we want to obtain an estimate

$$
\sup _{B_{r}}|f| \leqslant \sup _{K}|f|^{\alpha}
$$

for any $f \in A\left(B_{R}\right)$. It follows from the remark in the previous section that this inequality holds for some $\alpha$ if and only if $K$ is not a pluri-polar set and $\alpha$ can be estimated in terms of the (pluri-)capacity of $K$. We will use the fact that $K$ is a subset of $\mathbb{R}^{n}$ and get a condition on $K$ in real-analytic terms.

Consider the Hausdorff dimension of $K$. We will use the definition of dimension through the Riesz capacities and refer the reader to $\S 5.3$ for the details. It is clear that an $(N-1)$-dimensional subset $\left\{x=\left(x_{1}, \ldots, x_{N}\right) \in B_{R}, x_{1}=0\right\}$ is not a uniqueness set for 
the class $A\left(B_{R}\right)$. On the other hand, if $\operatorname{dim} K>N-1$, then $K$ is not pluri-polar and (3.1) holds. In fact, for this case $C_{s}(K)>0$ for some $s>N-1$. Then for almost all $b \in \mathbb{S}^{N-1}$ we have

$$
\mathcal{H}^{N-1}\left\{a \in b^{\perp}: C_{s-N+1}\left(K \cap l_{a, b}\right)>0\right\}>0,
$$

where $l_{a, b}=\{x=a+t b, t \in \mathbb{R}\}$ (see [14, Theorem 10.8]). Thus $l_{a, b} \cap K$ is not a polar set in the complex plane $L_{a, b}=\{z=a+\lambda b, \lambda \in \mathbb{C}\}$. Suppose that $K \subset\{u=-\infty\}$ for some pluri-subharmonic function $u$. Then for $b \in \mathbb{S}^{N-1}$ and $a \in b^{\perp}$ the restriction of $u$ to $L_{a, b}$ is equal to $-\infty$ identically provided that $C_{s-N+1}\left(K \cap l_{a, b}\right)>0$. Thus for almost all $b \in \mathbb{S}^{N-1}$ the restriction of $u$ on $b^{\perp}$ is equal to $-\infty$ on a set of positive measure. Since $u$ is pluri-subharmonic this implies $u=-\infty$ on $b^{\perp}$ for almost all $b \in \mathbb{S}^{N-1}$. And, finally, $u=-\infty$ identically.

Our aim is to get a quantitative estimate (3.1) for the case $\operatorname{dim} K>N-1$. We fix $\delta>0$ and assume that $K$ has positive Riesz $(N-1+\delta)$-capacity.

\subsection{Estimates for functions in $A(B)$}

First we prove inequality (3.1) under the assumption that $K$ has positive $N$ dimensional Lebesgue measure.

Lemma 3.1. Suppose that $K \subset B_{r}, 4 r<R<1$, and $m=m(K)>0$. Then (3.1) is satisfied for any $f \in A\left(B_{R}\right)$ with $\alpha=\alpha(r, N, m)$.

Proof. We fix a point $a \in B_{r}$ and consider all lines passing through $a$. We can find at least one line $l$ such that $|K \cap l|>c(N) r^{1-N} m$. In fact,

$$
m=m(K)=\int \chi_{K}(x) \mathrm{d} m(x)=\int_{\mathbb{S}^{N-1}} \int_{0}^{2 r} t^{N-1} \chi_{K}\left(a+t x^{\prime}\right) \mathrm{d} t \mathrm{~d} s\left(x^{\prime}\right),
$$

and there exists $x^{\prime} \in \mathbb{S}^{N-1}$ such that

$$
\begin{aligned}
\left|K \cap\left\{a+s x^{\prime}\right\}\right| & =\int_{0}^{2 r} \chi_{K}\left(a+t x^{\prime}\right) \mathrm{d} t \\
& \geqslant(2 r)^{1-N} \int_{0}^{2 r} t^{N-1} \chi_{K}\left(a+t x^{\prime}\right) \mathrm{d} t \geqslant c(N) r^{1-N} m .
\end{aligned}
$$

We take $l$ to be $\left\{a+s x^{\prime}: s \in \mathbb{R}\right\}$. Now let $2 \tilde{r}=\left|B_{r} \cap l\right|<2 r$. We apply Corollary 2.3 replacing $r, R$ and $K$ by $\tilde{r}, \sqrt{R^{2}-r^{2}+\tilde{r}^{2}}$ and $K \cap l$. Denote by $\tilde{f}$ the extension of $f \mid l$ to the complex ball of radius $\sqrt{R^{2}-r^{2}+\tilde{r}^{2}}$, then we have

$$
|f(a)| \leqslant \sup _{B_{\tilde{r}}}|\tilde{f}| \leqslant \sup _{K \cap l}|f|^{\alpha}
$$

where

$$
\alpha \geqslant \frac{1}{8}\left(\ln \frac{c(N) r^{N}}{m(K)}\right)^{-1}
$$

This completes the proof. 
We finish this section by proving the Key Lemma formulated in $\S 1$.

Lemma 3.2. Let $K \subset B_{r}, 4 r<R<1$, and let $\mathcal{C}(K)=C_{N-1+\delta}(K)>0$. Then (3.1) holds with

$$
\alpha=\alpha\left(N, \delta, r^{1-N-\delta} \mathcal{C}(K)\right) .
$$

Proof. Applying Lemma 5.3 to $K$ we can find the line $l$ such that

$$
\mathcal{H}^{N-1}\left\{a \in l^{\perp}: C_{\delta}\left(K \cap l_{a}\right) \geqslant k(N) r^{1-N} \mathcal{C}(K)\right\} \geqslant c_{1}(N) r^{-\delta} \mathcal{C}(K) .
$$

We denote by $P_{l}$ the orthogonal projection of $\mathbb{R}^{N}$ onto $l^{\perp}$ and by $L$ the set of $a \in l^{\perp}$ such that $C_{\delta}\left(K \cap l_{a}\right) \geqslant k(N) r^{1-N} \mathcal{C}(K)$. Then, we apply Theorem 2.1 to each set $K \cap l_{a}$ and use a simple estimate $C_{0}\left(K \cap l_{a}\right) \geqslant\left(C_{\delta}\left(K \cap l_{a}\right)\right)^{1 / \delta}$ (see (5.4) below). We get

$$
|f(x)| \leqslant \sup _{K}|f|^{\alpha} \quad \text { for any } x \in B_{r} \cap P_{l}^{-1}(L),
$$

where

$$
\alpha \geqslant \frac{1}{8 \delta}\left(\ln \frac{c(N) r^{N-1+\delta}}{\mathcal{C}(K)}\right)^{-1}
$$

Now we consider the hyperplanes $\left\{l^{\perp}+h\right\}$, where $h \in l,|h|^{2}<c_{3}(N) r^{-N+3-\delta} \mathcal{C}(K)$. Each of them intersects $P_{l}^{-1}(L) \cap B_{r}$ and, provided that $c_{3}(N)$ is small enough, we can estimate the measure of $E_{h}=\left(l^{\perp}+h\right) \cap B_{r} \cap P_{l}^{-1}(L)$,

$$
\mathcal{H}^{N-1}\left(E_{h}\right) \geqslant c_{4}(N) r^{-\delta} \mathcal{C}(K) .
$$

On $E_{h}$ estimate (3.2) is valid and, applying Lemma 3.1 to each $(N-1)$-dimensional set $E_{h}$, we get

$$
|f(x)| \leqslant \sup _{K}|f|^{\alpha}, \quad \text { whenever } \operatorname{dist}\left(x, l^{\perp}\right) \leqslant \sqrt{c_{3}(N) r^{-N+3-\delta} \mathcal{C}(K)},
$$

where

$$
\alpha \geqslant \frac{1}{64 \delta}\left(\ln \frac{c(N) r^{N-1+\delta}}{\mathcal{C}(K)}\right)^{-2}
$$

Finally,

$$
m\left\{x \in B_{r}: \operatorname{dist}\left(x, l^{\perp}\right) \leqslant c(N) \sqrt{r^{-N+3-\delta} \mathcal{C}(K)}\right\} \geqslant c(N) \sqrt{r^{N+1-\delta} \mathcal{C}(K)}
$$

and, using Lemma 3.1 once again, we have

$$
\sup _{B_{r}}|f| \leqslant \sup _{K}|f|^{\alpha}
$$

where

$$
\alpha \geqslant \frac{1}{256 \delta}\left(\ln \frac{c(N) r^{N-1+\delta}}{\mathcal{C}(K)}\right)^{-3}
$$

This completes the proof of the theorem. We obtained an explicit estimate for $\alpha$, but it looks too complicated to be used. 


\section{Prolongation of smallness for factors of the Laplacian}

\subsection{Definition and examples}

Let $A$ be a first-order linear differential operator with constant coefficients defined on vector functions, $A=\sum_{j=1}^{n} A_{j} \partial_{j}$, where $A_{j}$ are constant matrices. The system of equations

$$
(A F=) \sum_{j=1}^{n} A_{j} \frac{\partial F}{\partial x_{j}}=0
$$

is called a generalized Cauchy-Riemann (GCR) system if for any solution $F$ to this system, $F: \mathbb{R}^{n} \rightarrow \mathbb{R}^{m}, F=\left(f_{1}, \ldots, f_{m}\right)$, all its components $f_{1}, f_{2}, \ldots, f_{m}$ are harmonic (see [19]). (We suppose that $F$ is well defined and satisfies (4.1) in an open subset of $\mathbb{R}^{n}$.)

Definition 4.1. A GCR system (4.1) is said to be a factor of the Laplacian if there exists a first-order differential operator $B=\sum_{j=1}^{n} B_{j} \partial_{j}$ with constant coefficients such that $B A=\Delta$.

Let us remark that $B A=\Delta$ means that

$$
B_{j} A_{j}=I_{m}, \quad j=1, \ldots, n, \quad \text { and } \quad B_{j} A_{k}+B_{k} A_{j}=0, \quad 1 \leqslant j<k \leqslant n,
$$

where $I_{m}$ is the identity matrix.

Example 4.2. The simplest GCR system in $\mathbb{R}^{n}$ is

$$
\sum_{j=1}^{n} \frac{\partial f_{j}}{\partial x_{j}}=0, \quad \frac{\partial f_{j}}{\partial x_{k}}=\frac{\partial f_{k}}{\partial x_{j}}, \quad 1 \leqslant j<k \leqslant n .
$$

Clearly, it is a factor of the Laplacian. A solution to this system is locally the gradient of a harmonic function. In $\mathbb{R}^{2}=\mathbb{C},(4.2)$ is the classical Cauchy-Riemann system for $\left(f_{1},-f_{2}\right)$.

Example 4.3. We consider (non-homogeneous) differential forms in $\mathbb{R}^{n}$. Let $d$ be the exterior differential operator and $\delta$ the adjoint operator (see, for example, [7]). Then the system

$$
(d+\delta) \omega=0
$$

can be written as a system of first-order differential equations with constant coefficients and it is a factor of the Laplacian as $\Delta \omega=-(d \delta+\delta d) \omega=-(d+\delta)(d+\delta) \omega$. When $\omega=\sum_{j=1}^{N} f_{j} \mathrm{~d} x^{j}$ is a 1 -form, the system above coincides with (4.2).

Further examples and a description of rotationally invariant factors of the Laplacian can be found in [6] (see also [8] and references therein).

\subsection{Prolongation to an $(n-1)$-dimensional ball}

Now we are ready to prove the Main Result formulated in $\S 1$. First we shall apply the results of $\S 3$. In order to do this we need the following classical lemma. It can be found in textbooks on harmonic functions (see, for example, $[\mathbf{9}$, Chapter 1.5]). 
Lemma 4.4. Suppose that $h$ is a harmonic function in the ball $B_{R} \subset \mathbb{R}^{n}$ and $|h| \leqslant 1$ on $B_{R}, r=\frac{1}{3} R$. Then $h$ has an analytic continuation $\tilde{h}$ to $B_{r, \mathbb{C}}$ that is bounded by $c(n)$ and, for $z \in B_{r, \mathbb{C}}$,

$$
\left|\tilde{h}(z)-T_{k} \tilde{h}(z)\right| \leqslant c(n) \rho^{k},
$$

where $T_{k} \tilde{h}$ is the Taylor polynomial for $\tilde{h}$ and $\rho<1$.

Let $F=\left(f_{1}, \ldots, f_{m}\right)$ be a solution to a GCR system $A F=0$ in $B_{R} \subset \mathbb{R}^{n}$ and $\sup _{B_{R}}|F| \leqslant 1$. And let $K$ be a subset of the hyperplane $\left\{x_{n}=0\right\}$ with $\mathcal{C}(K)=$ $C_{n-2+\delta}(K)>0$ for some $\delta>0$. To prove the main result we should estimate $\sup _{B_{t}}|F|$ (where $t<R$ ) in terms of $\sup _{K}|F|$. We may assume that $K \subset B_{r}$, where $r<\frac{1}{12} R$. The first step is to estimate $|F|$ on $B_{r} \cap\left\{x_{n}=0\right\}$.

Each component $f_{j}$ of $F$ is a harmonic function. Applying Lemma 4.4, we see that $f_{j}$ can be extended to a bounded analytic function in $B_{R / 3, \mathbb{C}}$. Let $B_{r}^{\prime}=B_{r} \cap\left\{x_{n}=0\right\}$. Theorem 3.2 implies

$$
\sup _{B_{r}^{\prime}}|F| \leqslant c(n)\left(\sup _{K}|F|\right)^{\alpha}
$$

where $\alpha=\alpha(r, n, \mathcal{C}(K), \delta)$.

\subsection{From hyperplane to a cylinder}

Suppose that $|F| \leqslant 1$ on $B_{R}$ and $|F| \leqslant \varepsilon$ on $K$, where $K$ is as above. Then by (4.3) we have $|F| \leqslant c(n) \varepsilon^{\alpha}$ on $B_{r}^{\prime}$, where $12 r<R$. We shall extend this estimate to a ball in $\mathbb{R}^{n}$.

We consider analytic continuation $\tilde{F}$ of (all components of) $F$ that is obtained by the series expansion and diverges in $B_{r, \mathbb{C}}$. By Lemma 4.4 , we have $\sup _{B_{t, \mathbb{C}}^{\prime}}|\tilde{F}| \leqslant c(n) \varepsilon^{\alpha}$ for $t<\frac{1}{3} r$. Thus we can estimate the partial derivatives of $F$ with respect to the first $n-1$ variables. Letting $\lambda=\left(\lambda_{1}, \ldots, \lambda_{n-1}, 0\right)$, we have

$$
\left|\mathcal{D}^{\lambda} F\right| \leqslant c(n)|\lambda| !\left(\frac{2}{t}\right)^{|\lambda|} \varepsilon^{\alpha} \quad \text { on } B_{s}^{\prime}, \quad s<\frac{1}{2} t .
$$

To get estimates for the partial derivatives with respect to $x_{n}$, note that $F$ (with all its partial derivatives) satisfies a GCR system and all components of $F$ are harmonic functions. Thereby we have

$$
\left|\partial_{n} F\right|=\left|B_{n} A_{n} \partial_{n} F\right|=\left|B_{n}\left(-\sum_{1}^{n-1} A_{j} \partial_{j} F\right)\right| \leqslant c(A, n) \frac{2}{t} \varepsilon^{\alpha} \quad \text { on } B_{s}^{\prime} .
$$

Furthermore, the following estimates hold on $B_{s}^{\prime}$ :

$$
\left|\partial_{n}^{2 k} F\right|=\left|\left(-\sum_{1}^{n-1} \partial_{j}^{2}\right)^{k} F\right| \leqslant c(n) n^{k}(2 k) !\left(\frac{2}{t}\right)^{2 k} \varepsilon^{\alpha}
$$

and

$$
\left|\partial_{n}^{2 k+1} F\right|=\left|B_{n}\left(-\sum_{1}^{n-1} A_{j} \partial_{j}\left(-\sum_{1}^{n-1} \partial_{j}^{2}\right)^{k}\right) F\right| \leqslant c(n, A) n^{k}(2 k+1) !\left(\frac{2}{t}\right)^{2 k+1} \varepsilon^{\alpha} .
$$


Finally, for $x=\left(x^{\prime}, x_{n}\right)$ with $\left|x^{\prime}\right|<s$ and $x_{n}<t / 4 \sqrt{n}$, we have

$$
\left|F\left(x^{\prime}, x_{n}\right)\right|=\left|\sum_{0}^{\infty} \frac{1}{l !} \partial_{n}^{l} F\left(x^{\prime}, 0\right) x_{n}^{l}\right| \leqslant c(n, A) \varepsilon^{\alpha} .
$$

In particular, the last estimate is valid in the ball of radius $r / 12 \sqrt{n}$. Once again, using the three-balls theorem, we can replace the ball by any other that is compactly supported in $B_{R}$ and we are done.

\section{Some definitions and results from potential theory}

In this section results from potential theory that were used in the work are collected. Our main sources are $[\mathbf{1}, \mathbf{1 2}, \mathbf{1 4}]$.

\subsection{The Wiener and logarithmic capacities on the complex plane}

We follow Landkof's book [12, Chapter II, no. 15] to define the capacity of subsets of (the unit disc in) the complex plane. Let $E$ be a compact subset of the unit disc. Then the Wiener capacity of $E$ is

$$
\operatorname{cap}(E)=\left(\inf \left\{I(\mu)=\int_{E} \int_{E} \ln \frac{1}{|x-y|} \mathrm{d} \mu(x) \mathrm{d} \mu(y), \operatorname{supp}(\mu) \subset E, \mu(E)=1\right\}\right)^{-1},
$$

where the infimum is taken over all Radon measures $\mu$.

A compact subset $E$ of the unit disc is called polar if there exists a subharmonic function $u$ such that $\left.u\right|_{E}=-\infty ; E$ is a polar set if and only if $\operatorname{cap}(E)=0$. We shall also use the logarithmic capacity of $E$ defined by

$$
C_{0}(E)=\exp \left(-\operatorname{cap}(E)^{-1}\right)
$$

It is easy to see that $C_{0}$ is a non-negative monotone non-decreasing function of compact sets and the logarithmic capacity of a ball is given by $C_{0}\left(B_{r}\right)=r$ (see [12, Chapter II, nos 16, 17]). Another simple inequality we use is if $K$ is a compact subset of a line, then $C_{0}(K) \geqslant \frac{1}{4}|K|$, where $|K|$ is the linear measure of $K$.

\subsection{Chebyshev constants and transfinite diameter}

Denote by $\mathcal{P}_{m}$ the space of all polynomials of degree $m$ whose leading coefficient equals one. Then Chebyshev's constants for $E$ are given by

$$
\rho_{m}(E)=\min _{\mathcal{P}_{m}} \max _{E}\left|P_{m}(z)\right|^{1 / m} .
$$

Now, the $m$ th transfinite diameter of $E$ is

$$
d_{m}(E)=\max _{z_{j} \in E}\left(\prod_{0 \leqslant j<k \leqslant m}\left|z_{j}-z_{k}\right|\right)^{2 / m(m+1)}
$$


Then limits of both sequences $\left\{\rho_{m}\right\}$ and $\left\{d_{m}\right\}$ exist and are equal to the logarithmic capacity of $E$ (see $[\mathbf{1}$, Part I, $\S 7]$ ):

$$
\lim _{m \rightarrow \infty} \rho_{m}(E)=\lim _{m \rightarrow \infty} d_{m}(E)=C_{0}(E) .
$$

The following simple inequality was used:

$$
\rho_{m}(E) \geqslant C_{0}(E)
$$

In order to prove it let us take $P_{m}^{*} \in \mathcal{P}_{m}$ such that $\rho_{m}(E)=\max _{E}\left|P_{m}^{*}\right|^{1 / m}$. Then for any positive integer $n$ we have

$$
\rho_{m}(E)=\max _{E}\left|\left(P_{m}^{*}\right)^{n}\right|^{1 / m n} \geqslant \rho_{m n}(E) .
$$

As we know that $\lim _{m \rightarrow \infty} \rho_{m}(E)$ exists, we get (5.2) immediately.

\subsection{The Riesz capacities in $\mathbb{R}^{n}$ and the capacitary dimension}

Definition 5.1. Let $s>0$. The Riesz $s$-capacity of a set $A \subset \mathbb{R}^{N}$ is defined by

$$
C_{s}(A)=\sup \left\{I_{s}(\mu)^{-1}: \mu(A)=1\right\}
$$

where the supremum is taken over all Radon measures $\mu$ compactly supported on $A$, and

$$
I_{s}(\mu)=\iint|x-y|^{-s} \mathrm{~d} \mu(x) \mathrm{d} \mu(y) .
$$

Similarly, the logarithmic capacity of $A$ is defined by

$$
C_{0}(A)=\sup \left\{\exp \left(-\iint \ln \frac{1}{|x-y|} \mathrm{d} \mu(x) \mathrm{d} \mu(y)\right): \mu(A)=1\right\} .
$$

It is easy to check that $C_{s}\left(B_{r}\right)=c(s, n) r^{s}$ if $0<s<n$.

By the mean inequality, we have

$$
I_{s}(\mu) \geqslant \exp \left(s \iint \ln \frac{1}{|x-y|} \mathrm{d} \mu(x) \mathrm{d} \mu(y)\right)=\exp (s I(\mu)) .
$$

Thus,

$$
C_{s}(A) \leqslant C_{0}^{s}(A)
$$

where $C_{0}$ is the logarithmic capacity.

Definition 5.2. Let $A$ be a subset of $\mathbb{R}^{N}$. The capacitary dimension of $A$ is defined by

$$
\operatorname{dim}_{c}(A)=\sup \left\{s: C_{s}(A)>0\right\} .
$$

The remarkable fact is that the capacitary dimension coincides with the Hausdorff one (see [14, Chapter 8]). 


\subsection{Slicing}

Suppose that $A \subset R^{N}$ is such that $\mathcal{C}(A)=C_{N-1+\delta}(A)>0$ for some positive $\delta$. We consider intersections of $A$ and one-dimensional lines. Consider all lines passing through the origin, and for any such line $l$ and any $a \in l^{\perp}$ define $l_{a}=l+a$. The following inequality is used to reduce the multidimensional case to the one-dimensional one (see $[\mathbf{1 4}, \S 10.9]$ and $[\mathbf{1 7}])$ :

$$
\iint_{l^{\perp}} C_{\delta}\left(A \cap l_{a}\right) \mathrm{d} \mathcal{H}^{N-1}(a) \mathrm{d} \gamma_{N, 1}(l) \geqslant c_{0}(N) C_{N-1+\delta}(A),
$$

where $\gamma_{N, 1}$ is the measure on the manifold of all lines (in $\mathbb{R}^{N}$ ) passing through the origin which is induced by the surface measure on $\mathbb{S}^{N-1}$. This inequality implies the following lemma.

Lemma 5.3. There exist $k=k(N)$ such that for any $A \subset B_{r}$

$$
\mathcal{H}^{N-1}\left\{a \in l^{\perp}: C_{\delta}\left(A \cap l_{a}\right) \geqslant k r^{1-N} \mathcal{C}(A)\right\} \geqslant c_{1}(N) r^{-\delta} \mathcal{C}(A)
$$

is valid for at least one line $l$.

Proof. According to (5.5), there exists $l$ such that

$$
\int_{l^{\perp}} C_{\delta}\left(A \cap l_{a}\right) \mathrm{d} \mathcal{H}^{N-1}(a) \geqslant c_{0}(N) \mathcal{C}(A) .
$$

By the hypothesis $A \subset B_{r}, C_{\delta}\left(A \cap l_{a}\right) \leqslant C r^{\delta}$ and

$$
\mathcal{H}^{N-1}\left\{a \in l^{\perp}: A \cap l_{a} \neq \emptyset\right\} \leqslant c_{2}(N) r^{N-1} .
$$

For any $k>0$, we define

$$
E_{k}=\left\{a \in l^{\perp}: C_{\delta}\left(A \cap l_{a}\right) \geqslant k r^{1-N} \mathcal{C}(A)\right\} \quad \text { and } \quad H_{k}=\mathcal{H}^{N-1}\left(E_{k}\right) .
$$

Then we have

$$
\begin{aligned}
c_{0}(N) \mathcal{C}(A) & \leqslant \int_{l^{\perp}} C_{\delta}\left(A \cap l_{a}\right) \mathrm{d} \mathcal{H}^{N-1}(a) \\
& =\int_{E_{k}} C_{\delta}\left(A \cap l_{a}\right) \mathrm{d} \mathcal{H}^{N-1}(a)+\int_{l^{\perp} \backslash E_{k}} C_{\delta}\left(A \cap l_{a}\right) \mathrm{d} \mathcal{H}^{N-1}(a) \\
& \leqslant C r^{\delta} H_{k}+k r^{1-N} \mathcal{C}(A)\left(c_{2}(N) r^{N-1}-H_{k}\right) \\
& =\left(C r^{\delta}-k r^{1-N} \mathcal{C}(A)\right) H_{k}+c_{2}(N) k \mathcal{C}(A) .
\end{aligned}
$$

Taking $k$ small enough, we may assume that $C r^{\delta}-k r^{1-N} \mathcal{C}(A)>c(N) r^{\delta}>0$ (since $\left.A \subset B_{r}\right)$ and $c_{2}(N) k<c_{0}(N)$. Then we have $H_{k} \geqslant c_{1}(N) r^{-\delta} \mathcal{C}(A)$ and the lemma is proved.

Acknowledgements. The author was supported by a Norwegian Research Foundation postdoctoral fellowship. I thank Vladimir Eiderman, Victor Havin and Mikhail Sodin for useful discussions on the subject of the article. Thanks also go to Alexander Rashkovskii and Norman Levenberg, who pointed out the references on pluri-potential theory to me. I am very grateful to the referee, whose remarks and suggestions improved the final version of the article. 


\section{References}

1. H. Aiknwa And M. Essén, Potential theory-selected topics, Lecture Notes in Mathematics, vol. 1633 (Springer, 1996).

2. H. J. Alexander And B. A. TAYlor, Comparison of two capacities in $C^{n}$, Math. Z. 186 (1984), 407-417.

3. N. Arakelian and H. Shahgholian, Propagation of smallness for harmonic and analytic functions in arbitrary domains, Bull. Lond. Math. Soc. 31 (1999), 671-678.

4. E. BEDFord AND B. A. TAYlor, A new capacity for plurisubharmonic functions, Acta Math. 149 (1982), 1-40.

5. R. Brummelhuis, Three-spheres theorem for second order elliptic equations, J. Analyse Math. 65 (1995), 179-206.

6. K. M. Davis, E. J. Gilbert and R. A. Kunze, Elliptic differential operators in harmonic analysis, I, Generalized Cauchy-Riemann systems, Am. J. Math. 113 (1991), 75-116.

7. G. De Rham, Differentiable manifolds. Forms, currents, harmonic forms (Springer, 1984).

8. J. E. Gilbert And M. A. M. Murray, Clifford algebras and Dirac operators in harmonic analysis (Cambridge University Press, 1991).

9. W. K. Hayman And P. B. Kennedy, Subharmonic functions, vol. 1 (Academic, 1976).

10. J. KorevaAR AND J. L. H. MEYERS, Logarithmic convexity for supremum norms of harmonic functions, Bull. Lond. Math. Soc. 26 (1994), 353-362.

11. E. M. LAndis AND A. L. Gusarov, A variant of the three-balls theorem for solving an elliptic equation and a related theorem of Phragmén-Lindelöf type, Trudy Sem. Petrovsk. 8 (1982), 169-186 (in Russian).

12. N. S. LANDKOF, Foundations of modern potential theory (Springer, 1972).

13. N. Levenberg And B. A. TAYlor, Comparison of capacities in $C^{n}$, in Complex Analysis, Toulouse, 1983, pp. 162-172, Lecture Notes in Mathematics, vol. 1094 (Springer, 1984).

14. P. Mattila, Geometry of sets and measures in Euclidean spaces. Fractals and rectifiability, Cambridge Studies in Advanced Mathematics, vol. 44 (Cambridge University Press, 1995).

15. N. S. NADiRASHVILI, Estimation of the solutions of elliptic equations with analytic coefficients which are bounded on some set, Vestnik Mosk. Univ. Ser. I 2 (1979), 42-46 (in Russian).

16. N. S. NADIRASHVILI, Uniqueness and stability of continuation from a set to the domain of solution of an elliptic equation, Mat. Zametki 40 (1986), 218-225 (in Russian).

17. A. Sadullaev, Rational approximation and pluripolar sets, Mat. USSR Sb. 119(161) (1982), 96-118.

18. J. SiCIAK, Wiener's type sufficient conditions for regularity in $\mathbb{C}^{N}$, in Complex Analysis and Geometry, Paris, 199\%, pp. 39-46, Progress in Mathematics, vol. 188 (Birkhäuser, Basel, 2000).

19. E. STEIN AND G. WeISS, Introduction to Fourier analysis on Euclidean spaces (Princeton University Press, 1971).

20. S. Vessella, A continuous dependence result in the analytic continuation problem, Forum Math. 11 (1999), 695-703.

21. S. Vessella, Quantitative continuation from a measurable set of solutions of elliptic equations, Proc. R. Soc. Edinb. A 130 (2000), 909-923.

22. Th. H. Wolff, Counterexamples with harmonic gradients in $\mathbb{R}^{3}$, in Essays on Fourier analysis in honor of Elias M. Stein, pp. 321-384, Princeton Mathematics Series, vol. 42 (Princeton University Press, 1995). 